\title{
Increased fluoroquinolone resistance with time in Escherichia coli from $>$ I 7,000 patients at a large county hospital as a function of culture site, age, sex, and location

\author{
Lauren Becnel Boyd ${ }^{1}$, Robert L Atmar1,2, Graham L Randall ${ }^{3}$, \\ Richard J Hamill1,2,5, David Steffen ${ }^{4}$ and Lynn Zechiedrich*1,3
}

\begin{abstract}
Address: ${ }^{1}$ Department of Molecular Virology and Microbiology, Baylor College of Medicine, Houston, TX, 77030, USA, ${ }^{2}$ Department of Medicine, Baylor College of Medicine, Houston, TX, 77030, USA, ${ }^{3}$ Interinstitutional Program in Structural and Computational Biology and Molecular Biophysics, Baylor College of Medicine, Houston, TX, 77030, USA, ${ }^{4}$ Bioinformatics Resource Center, Baylor College of Medicine, Houston, TX, 77030, USA and ${ }^{5}$ Michael E. DeBakey Veterans Administration Medical Center, Houston, TX, 77030, USA

Email: Lauren Becnel Boyd - lb144335@bcm.edu; Robert L Atmar - ratmar@bcm.edu; Graham L Randall - grandall@bcm.edu; Richard J Hamill - richard.hamill@med.va.gov; David Steffen - steffen@bcm.edu; Lynn Zechiedrich* - elz@bcm.edu

* Corresponding author
\end{abstract}

Published: 15 January 2008

BMC Infectious Diseases 2008, 8:4 doi:10.1 186/147/-2334-8-4

This article is available from: http://www.biomedcentral.com/I47I-2334/8/4

(c) 2008 Boyd et al; licensee BioMed Central Ltd.

This is an Open Access article distributed under the terms of the Creative Commons Attribution License (http://creativecommons.org/licenses/by/2.0), which permits unrestricted use, distribution, and reproduction in any medium, provided the original work is properly cited.
Received: 2 October 2007

Accepted: 15 January 2008

\begin{abstract}
Background: Escherichia coli infections are common and often treated with fluoroquinolones. Fluoroquinolone resistance is of worldwide importance and is monitored by national and international surveillance networks. In this study, we analyzed the effects of time, culture site, and patient age, sex, and location on fluoroquinolone resistance in $E$. coli clinical isolates.
\end{abstract}

Methods: To understand how patient factors and time influenced fluoroquinolone resistance and to determine how well data from surveillance networks predict trends at Ben Taub General Hospital in Houston, TX, we used Perl to parse and MySQL to house data from antibiograms ( $n \cong$ 21,000 ) for E. coli isolated between 1999 to 2004 using Chi Square, Bonferroni, and Multiple Linear Regression methods.

Results: Fluoroquinolone resistance (i) increased with time; (ii) exceeded national averages by 2 to 4-fold; (iii) was higher in males than females, largely because of urinary isolates from male outpatients; (iv) increased with patient age; (v) was $3 \%$ in pediatric patients; (vi) was higher in hospitalized patients than outpatients; (vii) was higher in sputum samples, particularly from inpatients, than all other culture sites, including blood and urine, regardless of patient location; and (viii) was lowest in genital isolates than all other culture sites. Additionally, the data suggest that, with regard to susceptibility or resistance by the Dade Behring MicroScan system, a single fluoroquinolone suffices as a "surrogate marker" for all of the fluoroquinolone tested.

Conclusion: Large surveillance programs often did not predict $E$. coli fluoroquinolone resistance trends at a large, urban hospital with a largely indigent, ethnically diverse patient population or its affiliated community clinics. 


\section{Background}

E. coli is the most common etiologic agent of infections caused by Gram-negative bacilli, and these infections routinely are treated with fluoroquinolones, some of the most-frequently prescribed antibiotic classes [1]. National and international surveillance networks track the frequency of susceptibility to antimicrobial agents, including the fluoroquinolones. Some fluoroquinolone data, such as that showing that males are more likely than females to have resistant isolates, reveal clear trends [2-4]. Other data from these networks can vary. For example, one study uncovered that younger patient age was associated with increased likelihood of having a ciprofloxacin non-susceptible isolate [4], but another report of urinary E. coli isolates found that resistance was highest in patients $\geq 65$ years of age [5]. Low numbers of isolates from each participating hospital, variations in patient populations, and differences in geographical regions of these hospitals may play a role in the variation in the data. Large-scale, local studies, therefore, are required to understand drug resistance in a given community.

\section{Methods}

We analyzed the effects of patient factors on fluoroquinolone resistance over time at Ben Taub General Hospital, a 578 bed, acute-care, county hospital that serves a mostly Hispanic and African-American patient population in Houston, Texas. The hospital microbiology laboratory also provides service to twelve community health centers across Harris County, Texas. This retrospective study differs from surveillance network studies in that data from thousands of E. coli isolates from a single hospital laboratory were analyzed simultaneously. Antibiotic susceptibilities were determined using the Dade Behring MicroScan system (Sacramento, CA, USA) according to Clinical Laboratory Standards Institute (CLSI) guidelines [6]. Data from all E. coli antibiograms from July 1, 1999 to December 31, 2004 ( $\mathrm{n} \cong 21,000)$ were parsed with Perl and imported into a MySQL database (Uppsala, Sweden). All database queries only included information from the first isolate for each patient $(\mathrm{n} \cong 17,000)$. Female patients outnumbered males 3.5 to 1 , with age ranging from 0.01 to 103 years (average females $=39.5 \pm 20.6$, males $=41.9 \pm$ 24.9). Ciprofloxacin, gatifloxacin, levofloxacin, norfloxacin, and ofloxacin were included among the 25 different antibiotics in the antibiograms, although gatifloxacin has since lost approval for systemic, but not ophthalmic, use. Chi square analysis and the Bonferroni correction were used to analyze all the data, and $P \leq 0.01$ (99\% confidence interval) was required for statistical significance. We also determined the odds ratio of resistance to susceptibility with a multiple logistic regression equation using SPSS (Chicago, IL, USA).

\section{Results and Discussion}

\section{Fluoroquinolone resistance increased with time}

Surveillance networks reported that $\sim 5 \%$ of United States isolates were fluoroquinolone resistant [2-4,7]. Data averaged from 1999 - 2004 for each fluoroquinolone, however, showed that resistance exceeded the previously reported values by 2 - to 4 -fold (Fig. 1A). As expected (because ofloxacin is a racemic mixture of levofloxacin and its inactive enantiomer), the prevalence of levofloxacin and ofloxacin non-susceptibility was nearly identical $(\sim 10 \%)$. The frequency of non-susceptibility to levofloxacin and ofloxacin was approximately half that of ciprofloxacin, gatifloxacin, or norfloxacin $(\sim 18 \%)$, and the latter three were statistically indistinguishable (Fig. 1A). Regardless of year or drug, $<2 \%$ of isolates were intermediate resistant (Figs. 1A, 1B, and 1C); data from intermediate resistant isolates were not included in subsequent analyses. When data from all fluoroquinolones tested in a given year were combined, the frequency of non-susceptibility increased with time from $\sim 6 \%$ to almost 25\% (Fig. 1B). Binary regression showed that, with each passing month, the odds of having a resistant isolate significantly increased 1.024 -fold $(P<0.001)$. Two other studies from the region found a similar high resistance frequency. One found $\sim 13 \%$ of E. coli from febrile, neutropenic cancer patients undergoing chemotherapy at Houston's M.D. Anderson Cancer Center were ciprofloxacin resistant [8]. Fluoroquinolone prophylaxis is common in these patients, so one might expect a higher incidence of resistance than in the general population. The second found that $\sim 20 \%$ of the 59 isolates from the urine of outpatients in the "West South Central" (Arkansas, Louisiana, Oklahoma, and Texas) area were resistant to levofloxacin and ciprofloxacin [5]. Thus, it may be that fluoroquinolone resistance is significantly higher for patients in Texas than the rest of the nation.

It has been thought that fluoroquinolones exemplify a "class effect," such that when resistance mechanisms decrease susceptibility for one drug, they do so simultaneously for all [9]. Each mechanism, however, affects different fluoroquinolones to varying extents [10], and some mechanisms, such as Aac(6')-Ib-cr [11] and QepA [12], decrease susceptibility only to ciprofloxacin and norfloxacin. Thus, it was important to distinguish whether time or specific fluoroquinolone accounted for the resistance differences shown in Fig. 1A. Comparing fluoroquinolones for a single year (2002), in which ciprofloxacin, gatifloxacin, and levofloxacin were all tested routinely, revealed that the frequency of resistance for the three fluoroquinolones was statistically indistinguishable, 15\% (Fig. 1C). An independent test of whether there were any variations among the fluoroquinolones was to look for isolates that were "I" or "R" to one drug and "S" to another. When ciprofloxacin and gatifloxacin $(n=6,272)$, 

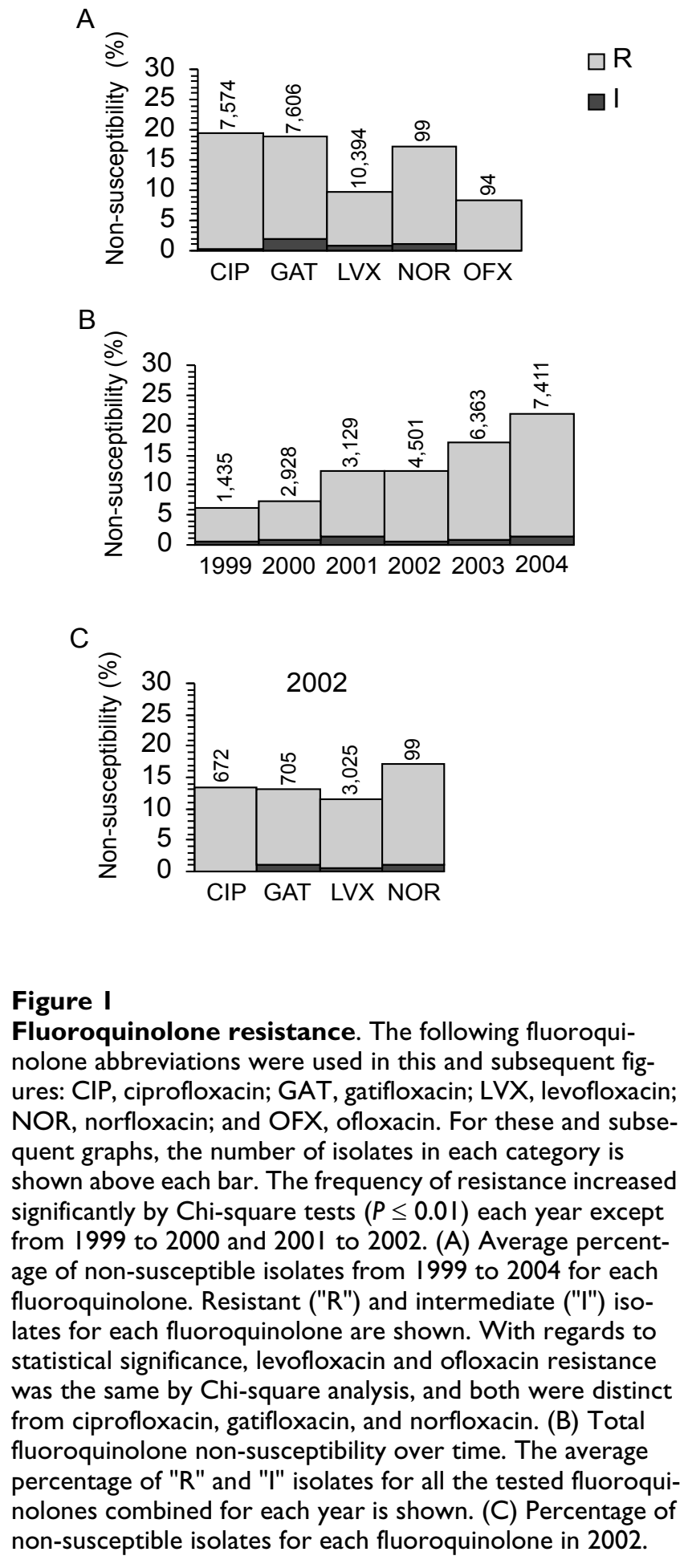

levofloxacin and ciprofloxacin $(\mathrm{n}=560)$, or levofloxacin and gatifloxacin $(n=582)$ were analyzed, $1-4 \%$ of isolates were non-susceptible to one drug and susceptible to the other. The small number of differentially susceptible isolates were equally likely to be non-susceptible to one drug while susceptible to another, and these few measurement differences likely represent the Dade MicroScan error rate [13].

To test whether time or specific fluoroquinolone accounted for the differences in Fig. 1A, we measured fluoroquinolone MICs by the agar dilution method independently in our laboratory [6]. We quantified MICs in 242 representative isolates from Ben Taub that were collected over the duration of this study. When ciprofloxacin, gatifloxacin, levofloxacin, and norfloxacin MICs were compared, all isolates that had non-susceptible MICs to one fluoroquinolone had non-susceptible MICs for all four drugs. Thus, what initially appeared to be drug differences in Fig. 1A were, in fact, a consequence of which drugs were tested over time. Our data and others [14] suggest that the susceptibility status of at least ciprofloxacin, gatifloxacin, levofloxacin, and norfloxacin could be inferred from testing only one of these drugs as a "surrogate marker." Because of these data, we conclude that fluoroquinolones indeed exhibit a class effect with regard to susceptibility as measured by the Dade Behring MicroScan system, and we combine data for all fluoroquinolones in subsequent analyses.

\section{Variation in resistance in the hospital and at outpatient community clinics}

Unlike most antibiotics in the United States, ciprofloxacin resistance has not been reported to be higher in isolates from patients in the ICU than in inpatients and outpatients $[3,4,15]$. In our population, however, resistance ( $19 \%$ of $>4,000$ patients) in ICU and inpatient isolates (statistically indistinguishable from each other) occurred more frequently than in outpatients $(\sim 9 \%$ of $>12,500$ patients, $P<0.0001$; data not shown). Hospital outpatients were significantly more likely $(P<0.001)$ to have a resistant isolate $(11 \%)$ than outpatients who received care from the twelve community health centers ( $\sim 8 \%)$. Resistance at most community clinics was statistically indistinguishable from community outpatients as a whole, except for two clinics with significantly higher resistance ( $14 \%$, $P<0.01)$ and two other clinics where resistance was significantly lower $(\sim 3 \%, P<0.001)$. Outpatient community health centers serviced by the hospital microbiology laboratory provided care to all patients seeking treatment, with the exception of the clinic with the highest prevalence of resistance, which provides care for HIV-positive patients. Unlike the HIV clinic, patients at all other clinics did not have any known specific illness. These data show that the majority of outpatients had the same likelihood of having 
a resistant isolate, regardless of whether they received care in the community or at the hospital.

\section{Fluoroquinolone resistance as a function of culture site} Isolates from abscesses, bloodstream, exudate, fluids and wounds were not different from each other or from urine ( $10 \%$ resistant, Table 1). Genital isolates (all from females) were significantly less likely $(0.1 \%, P<0.0001)$ to be resistant than isolates from other sites. Sputum samples had the highest frequency $(\sim 30 \%)$ of fluoroquinolone resistance $(P=0.001)$, with resistance being significantly more likely in inpatients $(\sim 40 \%)$ than ICU $(\sim 20 \%)$ patients. Chronic bronchitis, pneumonia, or complications of emphysema (all of which are common in chronic obstructive pulmonary disease, or COPD, patients) are often treated with fluoroquinolones, which facilitate recovery in hospitalized patients $[16,17]$. Thus, it is possible that the resistant sputum isolates originated from patients suffering from COPD. Miscellaneous other culture sites (Table 1) were too infrequent to be included in statistical analyses; however, bone ( $50 \%$ resistant), bronchia $(\sim 30 \%)$, and catheter tip $(\sim 40 \%)$ isolates also had alarmingly high frequencies of resistance (Table 1).

\section{Fluoroquinolone resistance as a function of patient age}

We determined the percentage of isolates that were fluoroquinolone resistant in each 10-year age bracket up to 70 years of age. Because relatively few patients were older than 70 years, patients of age $71-103$ were grouped

Table I: Isolate culture sites as a function of susceptibility status

\begin{tabular}{|c|c|c|c|c|}
\hline & \multirow[b]{2}{*}{ Culture Site } & \multicolumn{3}{|c|}{ Number of isolates } \\
\hline & & $\mathbf{S}$ & $\mathbf{I}$ & $\mathbf{R}(\%)$ \\
\hline \multirow[t]{8}{*}{ Includeda } & Abscess & 388 & 7 & $51(11)$ \\
\hline & Blood & 864 & 16 & $175(17)$ \\
\hline & Exudate & 602 & 2 & $84(12)$ \\
\hline & Fluids & 273 & 2 & $36(12)$ \\
\hline & Genitals & 259 & 1 & $2(1)$ \\
\hline & Sputum & 271 & 4 & $128(32)$ \\
\hline & Urine & 12,382 & 197 & $\mathrm{I}, 46 \mathrm{I}(\mathrm{II})$ \\
\hline & Wounds & 237 & 1 & $32(12)$ \\
\hline \multirow[t]{11}{*}{ Not included } & Bone & 4 & 0 & $5(56)$ \\
\hline & Bone marrow & 2 & 0 & $0(0)$ \\
\hline & Bronchia & 13 & 0 & $6(32)$ \\
\hline & Catheter tip & 38 & 3 & $24(37)$ \\
\hline & Cerebrospinal fluid & 12 & 0 & $0(0)$ \\
\hline & Ear & 7 & 0 & $0(0)$ \\
\hline & Enteric & 2 & 0 & $0(0)$ \\
\hline & Eye & 26 & 0 & $5(16)$ \\
\hline & Miscellaneous Tissue & 37 & 0 & $9(20)$ \\
\hline & Respiratory Tract & 21 & 0 & $3(12)$ \\
\hline & Trachea & 33 & 0 & $4(I I)$ \\
\hline
\end{tabular}

aSubjected to statistical analyses. Culture sites with fewer than five "S," "I," or "R" isolates were not analyzed because of insufficient statistical power. together. We found, overall, that fluoroquinolone resistance increased significantly with patient age (Fig. 2; $P<$ 0.001 ), and that the odds of an isolate being resistant increased 1.027 -fold for each year of age $(P<0.0001)$. The increasing resistance with patient age was not associated with a specific patient location. Because decreased immune function and overall health are associated with advanced age, the increased occurrence of resistance in isolates from older patients may have resulted from more frequent fluoroquinolone exposure than that for younger patients.

Approximately $2 \%$ of $\leq 10$ year-old children had fluoroquinolone-resistant $E$. coli isolates. These children should have been naïve to fluoroquinolones, as the use of these antibiotics in pediatric patients generally is not recommended. To ascertain whether these children presented with the infections around the same time, which could indicate an outbreak or clonal spread, we determined when and where they had been treated. The majority of children with resistant isolates were outpatients, but no

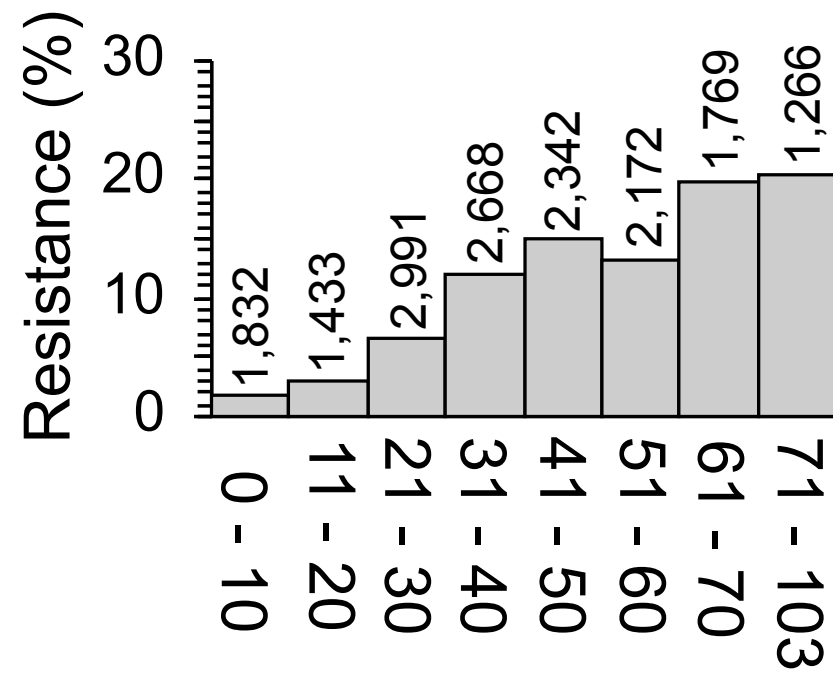

\section{Figure 2}

Fluoroquinolone resistance as a function of patient age. The percentage of resistant isolates is shown by age bracket. Patients $\geq 70$ years old were grouped. All data were analyzed by binary regression and Chi-square tests. With regards to statistical significance, overall, fluoroquinolone resistance increased with patient age. 
obvious temporal association was detected. Although the only licensed use of fluoroquinolones in children is treatment of post-inhalation anthrax exposure with ciprofloxacin, some compassionate use occurs in children suffering from serious infections, such as multidrug resistant infections [18]. In Houston, children with serious illness generally would be treated at Texas Children's Hospital, not Ben Taub General Hospital. Thus, most pediatric patients at Ben Taub, just as in the United States as a whole, should not have had prior fluoroquinolone exposure. Although our data did not control for previous antibiotic therapy, two previous studies that did also found that $\sim 2 \%$ of children harbored quinolone- or fluoroquinolone-resistant isolates $[19,20]$. Therefore, a small percentage of children may carry fluoroquinolone-resistant E. coli independently of prior fluoroquinolone use. The affected children may have acquired the fluoroquinolone-resistant isolates from a family member or in the community, although outbreaks of gram-negative bacteria are rarely reported [21]. However, because such sur- veillance efforts currently monitor the spread of virulent bacteria like E. coli O157:H7, less virulent strains that do not cause significant morbidity might be passed undetected from person to person.

\section{Increased resistance in urinary isolates from male outpatients}

Like previous studies $[3,4,22,23]$, males from Ben Taub between 1999 to 2004 were significantly more likely to have resistant isolates than females (Fig. 3A). We addressed whether patient location or culture site played a role. The percentage of male outpatients with resistant isolates was significantly higher $(P<0.001)$ than female outpatients (Fig. 3B). The sex difference was smaller, but still significant $(P=0.001)$ for inpatients, and no resistance difference between the sexes existed in isolates from ICU patients. Although for the majority of culture sites the sexes were indistinguishable, the frequency of resistance was significantly higher $(P<0.001)$ in urine cultures from males compared to females (Fig. 3C). Increased fluoro-
A

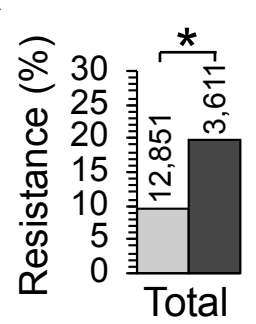

$\mathrm{B}$

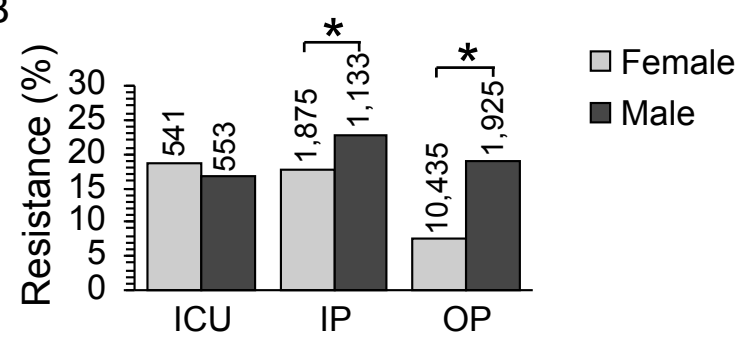

C

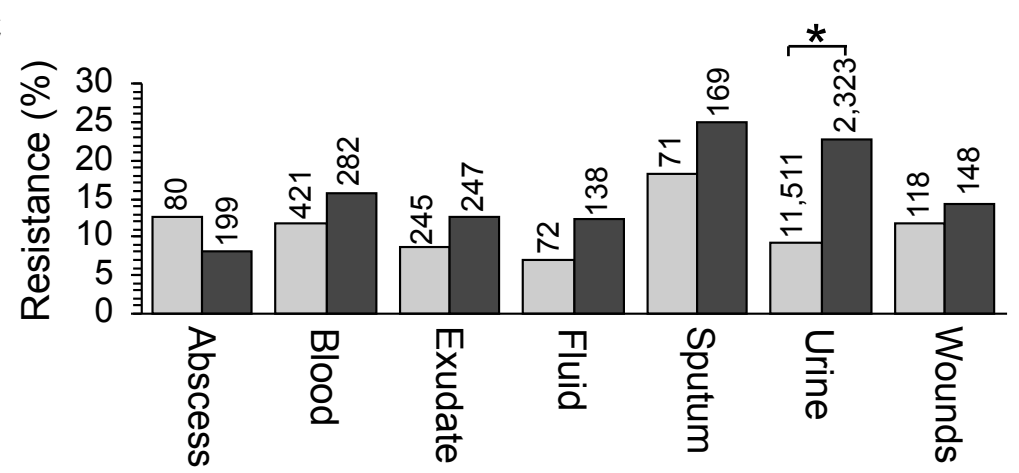

Figure 3

Fluoroquinolone resistance as a function of patient sex. Asterisks denote statistically significant differences. All data were analyzed by binary regression and Chi-square tests. (A) The percentage of resistant isolates is shown for female and male patients. (B) Fluoroquinolone resistance in female and male patients as a function of location. Because so few fluoroquinoloneresistant genital isolates existed, these data were excluded from analysis. (C) Fluoroquinolone resistance in female and male patients as a function of culture site. 
quinolone resistance in males, therefore, is attributable to isolates from the urine of outpatients. Unlike female urinary tract infections (UTIs), male UTIs are frequently complicated and are more likely to require prolonged antimicrobial therapy, potentially explaining the fluoroquinolone resistance discrepancies between the sexes $[24,25]$. Thus, differences in the type of UTI may impact the variation in resistance between the sexes. Our data cannot distinguish males with urinary tract infections from those with prostatitis. Fluoroquinolones are used to treat chronic prostatitis, even though they do not all readily penetrate the prostate $[26,27]$. Doses of fluoroquinolones that are less than the minimal inhibitory concentration (MIC) lead to selection of resistance mutations by a process known as "sub-inhibitory MIC effects" $[28,29]$. It is possible that such selection could play a role in the difference in resistance frequency between the sexes.

\section{Conclusion}

Fluoroquinolone resistance patterns are complicated, making it difficult to apply national data to clinical practice in any specific area. Studies that identify resistance trends on a local or regional scale ([30,31], this study) are more directly applicable to a given area and could help better guide prescribing practices of clinicians. In addition, it is important to disseminate such regional data, as they might be harbingers of trends that may spread to or be encountered in other regions in the future. This is particularly true for the fluoroquinolones, because their prescribing increased sharply since the 1990s in the United States [32] and likely in other countries worldwide as well. Finally, meta-analyses of regional studies might explain the variations in resistance trends from international surveillance networks and even predict such trends not only for E. coli, but also for other bacterial species, given the high conservation of the fluoroquinolone target topoisomerases across divergent species.

\section{Competing interests}

The author(s) declare that they have no competing interests.

\section{Authors' contributions}

LBB parsed the original antibiogram data using Perl, designed and implemented the MySQL database, performed statistical analyses, and wrote the manuscript. RLA obtained the antibiogram data and participated in the data analysis and editing of the manuscript. GLR helped with data analysis, performed binary regression statistical analysis, and edited the manuscript. RJH, DS, and LZ collaborated in the design, data interpretation, and editing of the manuscript. All authors read and approved the final manuscript.

\section{Acknowledgements}

We thank Barbara E. Murray, M.D.; Timothy G. Palzkill, Ph.D.; Joseph F. Petrosino, Ph.D.; Charles E. Stager, Ph.D.; and James Versalovic, M.D. Ph.D., for advice and Christopher H. Boyd; Daniel J. Catanese, Jr., Ph.D.; and Robert M. Ward for technical assistance. This work was funded by NIH Grant ROI Al054830 (ELZ), by the Pharmacoinformatics (NIH Grant T90 DK070 109; LSB) and the Computational Biology and Medicine (NLM Grant TI5 LM07093; GLR) Training Programs of the W. M. Keck Center for Computational and Structural Biology of the Gulf Coast Consortia, and in part by the Department of Veterans Affairs (RJH).

\section{References}

I. Linder JA, Huang FS, Steinman MA, Gonzalez R, Stafford RS: Fluoroquinolone prescribing in the United States: 1995 to 2002. Am J Med 2005, I I 8:259-268.

2. Jones ME, Draghi DC, Thornsberry C, Karlowsky JA, Sahm DF, Wenzel RP: Emerging resistance among bacterial pathogens in the intensive care unit--a European and North American Surveillance study (2000-2002). Ann Clin Microbiol Antimicrob 2004, 3:14.

3. Karlowsky JA, Kelly LJ, Thornsberry C, Jones ME, Evangelista AT, Critchley IA, Sahm DF: Susceptibility to fluoroquinolones among commonly isolated Gram-negative bacilli in 2000: TRUST and TSN data for the United States. Tracking Resistance in the United States Today. The Surveillance Network. Int J Antimicrob Agents 2002, I 9(I):2I-31.

4. Stelling JM, Travers K, Jones RN, Turner PJ, O'Brien TF, Levy SB: Integrating Escherichia coli antimicrobial susceptibility data from multiple surveillance programs. Emerg Infect Dis 2005, I I (6):873-882.

5. Zhanel GG, Hisanaga TL, Laing NM, DeCorby MR, Nichol KA, Weshnoweski B, Johnson J, Noreddin A, Low DE, Karlowsky JA, Hoban DJ: Antibiotic resistance in Escherichia coli outpatient urinary isolates: final results from the North American Urinary Tract Infection Collaborative Alliance (NAUTICA). Int J Antimicrob Agents 2006, 27(6):468-475.

6. NCCLS: Performance standards for antimicrobial susceptibility testing: ninth informational supplement. National Committee for Clinical Laboratory Standards 2002.

7. Mathai $D$, Jones RN, Pfaller MA: Epidemiology and frequency of resistance among pathogens causing urinary tract infections in 1,510 hospitalized patients: a report from the SENTRY Antimicrobial Surveillance Program (North America). Diagn Microbiol Infect Dis 200I, 40(3): I 29-I 36.

8. Jacobson K, Rolston K, Elting L, LeBlanc B, Whimbey E, Ho DH: Susceptibility surveillance among gram-negative bacilli at a cancer center. Chemotherapy 1999, 45(5):325-334.

9. Hopkins KL, Davies RH, Threlfall EJ: Mechanisms of quinolone resistance in Escherichia coli and Salmonella: recent developments. Int J Antimicrob Agents 2005, 25(5):358-373.

10. Jacoby GA: Mechanisms of resistance to quinolones. Clin Infect Dis 2005, 4I Suppl 2:SI 20-6.

II. Robicsek A, Jacoby GA, Hooper DC: The worldwide emergence of plasmid-mediated quinolone resistance. Lancet Infect Dis 2006, 6( ( 1 0):629-640.

12. Perichon B, Courvalin P, Galimand M: Transferable Resistance to Aminoglycosides by Methylation of GI405 in 16S rRNA and to Hydrophilic Fluoroquinolones by QepA-Mediated Efflux in Escherichia coli. Antimicrob Agents Chemother 2007, 5 I:2464-2469.

13. Steward CD, Stocker SA, Swenson JM, O'Hara CM, Edwards JR, Gaynes RP, McGowan JE Jr., Tenover FC: Comparison of agar dilution, disk diffusion, MicroScan, and Vitek antimicrobial susceptibility testing methods to broth microdilution for detection of fluoroquinolone-resistant isolates of the family Enterobacteriaceae. J Clin Microbiol I 999, 37(3):544-547.

14. Jones RN, Pfaller MA: Can antimicrobial susceptibility testing results for ciprofloxacin or levofloxacin predict susceptibility to a newer fluoroquinolone, gatifloxacin?: Report from The SENTRY Antimicrobial Surveillance Program (1997-99). Diagn Microbiol Infect Dis 200I, 39(4):237-243.

15. Fridkin SK, Edwards JR, Tenover FC, Gaynes RP, McGowan JE Jr.: Antimicrobial resistance prevalence rates in hospital antibi- 
ograms reflect prevalence rates among pathogens associated with hospital-acquired infections. Clin Infect Dis 200I, 33(3):324-330.

16. Saint $S$, Bent S, Vittinghoff E, Grady D: Antibiotics in chronic obstructive pulmonary disease exacerbations. A meta-analysis. Jama 1995, 273( I 2):957-960.

17. Hunter MH, King DE: COPD: management of acute exacerbations and chronic stable disease. Am Fam Physician 200I, 64(4):603-6I2.

18. The use of systemic fluoroquinolones. Pediatrics 2006 I I 8(3): I 287-1292.

19. Hannah EL, Angulo FJ, Johnson JR, Haddadin B, Williamson J, Samore $\mathrm{MH}$ : Drug-resistant Escherichia coli, Rural Idaho. Emerg Infect Dis 2005, I I ( I 0): 1614-1617.

20. Qin X, Razia Y, Johnson JR, Stapp JR, Boster DR, Tsosie T, Smith DL, Braden CR, Gay K, Angulo FJ, Tarr PI: Ciprofloxacin-resistant gram-negative bacilli in the fecal microflora of children. Antimicrob Agents Chemother 2006, 50( I 0):3325-3329.

21. Canton R, Coque TM, Baquero F: Multi-resistant Gram-negative bacilli: from epidemics to endemics. Curr Opin Infect Dis 2003 , 16(4):315-325.

22. Karlowsky JA, Kelly LJ, Thornsberry C, Jones ME, Sahm DF: Trends in antimicrobial resistance among urinary tract infection isolates of Escherichia coli from female outpatients in the United States. Antimicrob Agents Chemother 2002, 46(8):2540-2545

23. Sahm DF, Thornsberry C, Mayfield DC, Jones ME, Karlowsky JA: Multidrug-resistant urinary tract isolates of Escherichia coli: prevalence and patient demographics in the United States in 2000. Antimicrob Agents Chemother 200I, 45(5): I 402-I 406.

24. Alos JI, Serrano MG, Gomez-Garces JL, Perianes J: Antibiotic resistance of Escherichia coli from community-acquired urinary tract infections in relation to demographic and clinical data. Clin Microbiol Infect 2005, II (3):199-203.

25. Hummers-Pradier E, Koch M, Ohse AM, Heizmann WR, Kochen MM: Antibiotic resistance of urinary pathogens in female general practice patients. Scand J Infect Dis 2005, 37(4):256-26I.

26. Naber CK, Steghafner M, Kinzig-Schippers M, Sauber C, Sorgel F, Stahlberg HJ, Naber KG: Concentrations of gatifloxacin in plasma and urine and penetration into prostatic and semina fluid, ejaculate, and sperm cells after single oral administrations of $\mathbf{4 0 0}$ milligrams to volunteers. Antimicrob Agents Chemother 200I, 45(I):293-297.

27. Wagenlehner FM, Naber KG: Fluoroquinolone Antimicrobial Agents in the Treatment of Prostatitis and Recurrent Urinary Tract Infections in Men. Curr Infect Dis Rep 2005, 7(I):9-16.

28. Gillespie SH, Basu S, Dickens AL, O'Sullivan DM, McHugh TD: Effect of subinhibitory concentrations of ciprofloxacin on Mycobacterium fortuitum mutation rates. J Antimicrob Chemother 2005, 56(2):344-348.

29. Licata L, Smith CE, Goldschmidt RM, Barrett JF, Frosco M: Comparison of the postantibiotic and postantibiotic sub-MIC effects of levofloxacin and ciprofloxacin on Staphylococcus aureus and Streptococcus pneumoniae. Antimicrob Agents Chemother 1997, 4 I(5):950-955.

30. Zervos MJ, Hershberger E, Nicolau DP, Ritchie DJ, Blackner LK Coyle EA, Donnelly AJ, Eckel SF, Eng RH, Hiltz A, Kuyumjian AG, Krebs W, McDaniel A, Hogan P, Lubowski TJ: Relationship between fluoroquinolone use and changes in susceptibility to fluoroquinolones of selected pathogens in 10 United States teaching hospitals, 199|-2000. Clin Infect Dis 2003, 37(12): 1643-1648.

31. Saurina G, Quale JM, Manikal VM, Oydna E, Landman D: Antimicrobial resistance in Enterobacteriaceae in Brooklyn, NY: epidemiology and relation to antibiotic usage patterns. Antimicrob Chemother 2000, 45(6):895-898.

32. Neuhauser MM, Weinstein RA, Rydman R, Danziger LH, Karam G, Quinn JP: Antibiotic resistance among gram-negative bacilli in US intensive care units: implications for fluoroquinolone use. Jama 2003, 289(7):885-888.

\section{Pre-publication history}

The pre-publication history for this paper can be accessed here: http://www.biomedcentral.com/1471-2334/8/4/prepub
Publish with Biomed Central and every scientist can read your work free of charge

"BioMed Central will be the most significant development for disseminating the results of biomedical research in our lifetime. "

Sir Paul Nurse, Cancer Research UK

Your research papers will be:

- available free of charge to the entire biomedical community

- peer reviewed and published immediately upon acceptance

- cited in PubMed and archived on PubMed Central

- yours - you keep the copyright
BioMedcentral 